\title{
Synthesis and Biological Evaluation of Iodinated Fidaxomicin Antibiotics
}

Dorst, Andrea ; Shchelik, Inga S ; Schäfle, Daniel ; Sander, Peter ; Gademann, Karl

\begin{abstract}
Fidaxomicin (1, tiacumicin B, lipiarmycin A3) is a marketed antibiotic that is used in the treatment of Clostridium difficile infections. Based on the analysis of a cryo-EM structure of fidaxomicin binding to its target enzyme (RNA-polymerase), a cation- interaction of the aromatic moiety with an arginine residue was identified. Therefore, the variation of the substituents and concurrently changing the electronic properties of the aryl moiety represents an interesting strategy in the search for new fidaxomicin analogs. Herein, we report the first semisynthetic access to new fidaxomicin analogs with varying halogen substituents through a Pd-catalyzed hydrodechlorination reaction. Subsequent iodination gave access to the first iodo-fidaxomicin derivatives, which matched or improved antibacterial properties compared to fidaxomicin against Mycobacterium tuberculosis and Staphylococcus aureus ATCC 29213.
\end{abstract}

DOI: https://doi.org/10.1002/hlca.202000130

Posted at the Zurich Open Repository and Archive, University of Zurich

ZORA URL: https://doi.org/10.5167/uzh-190540

Journal Article

Accepted Version

Originally published at:

Dorst, Andrea; Shchelik, Inga S; Schäfle, Daniel; Sander, Peter; Gademann, Karl (2020). Synthesis and Biological Evaluation of Iodinated Fidaxomicin Antibiotics. Helvetica Chimica Acta, 103(9):e2000130.

DOI: https://doi.org/10.1002/hlca.202000130 


\title{
Synthesis and Biological Evaluation of lodinated Fidaxomicin Antibiotics
}

\author{
Andrea Dorst, ${ }^{\mathrm{a}}$ Inga S. Shchelik, ${ }^{\mathrm{a}}$ Daniel Schäfle, ${ }^{\mathrm{b}}$ Peter Sander, ${ }^{\mathrm{b}, \mathrm{c}}$ and Karl Gademann*,a \\ a Department of Chemistry, University of Zurich, Winterthurerstrasse 190, CH-8057 Zurich, Switzerland, e-mail: karl.gademann@chem.uzh.ch \\ b Institute of Medical Microbiology, University of Zurich, Gloriastrasse 28/30, CH-8006 Zurich, Switzerland \\ c National Center for Mycobacteria, University of Zurich, Gloriastrasse 28/30, CH-8006 Zurich, Switzerland
}

Fidaxomicin (1, tiacumicin B, lipiarmycin A3) is a marketed antibiotic that is used in the treatment of $C$. difficile infections. Based on the analysis of a cryoEM structure of fidaxomicin binding to its target enzyme (RNA-polymerase), a cation- $\pi$ interaction of the aromatic moiety with an arginine residue was identified. Therefore, the variation of the substituents and concurrently changing the electronic properties of the aryl moiety represents an interesting strategy in search for new fidaxomicin analogs. Herein, we report the first semisynthetic access to new fidaxomicin analogs with varying halogen substituents via a Pd-catalyzed hydrodechlorination reaction. Subsequent iodination gave access to the first iodo-fidaxomicin derivatives, which matched or improved antibacterial properties compared to fidaxomicin against Mycobacterium tuberculosis and Staphylococcus aureus ATCC 29213.

Keywords: fidaxomicin • antibiotics • iodination • semisynthesis $\bullet$ natural products

\section{Introduction}

Fidaxomicin (1, tiacumicin B, lipiarmycin A3) constitutes a glycosylated macrocyclic lactone first discovered in 1972 in a culture of $A$. deccanensis ${ }^{[1-}$ ${ }^{3]}$ and later re-isolated several times over the years. ${ }^{[4-11]}$ This complex natural product has been introduced in the clinic and was reported to display superior properties compared to vancomycin and metronidazole. ${ }^{[12]}$ Fidaxomicin shows a narrower spectrum of activity and it is particularly active against $C$. difficile, allowing the normal gut flora to recover. ${ }^{[13]}$ Furthermore, fidaxomicin is poorly absorbed when orally administered, which reduces its side effects. ${ }^{[14]}$

Over the last decades, the number of strains exhibiting reduced susceptibility and even resistance to antibiotics is increasing steadily in the clinic, ${ }^{[15]}$ which led various institutions such as governmental agencies and the World Health Organization to develop plans for action. ${ }^{[16][17]}$ In the past, natural products were successfully used as lead structures for the development of new drugs with improved activities and properties. ${ }^{[18-20]}$ Therefore, the synthesis of novel fidaxomicin derivatives can provide avenues to successfully combat antibiotic resistance ${ }^{[21]}$ and consequently access to various fidaxomicin derivatives will be needed in future.

Fidaxomicin is an RNA-Polymerase (RNAP) inhibitor with a unique mode of action. ${ }^{[22-28]}$ The recent disclosure of cryo-EM structures ${ }^{[29,30]}$

\section{Previous work:}

Abbott Laboratories:[42,43] $\mathrm{Cl} \rightarrow \mathrm{Br}$
sustitution

D. aurantiacum in media

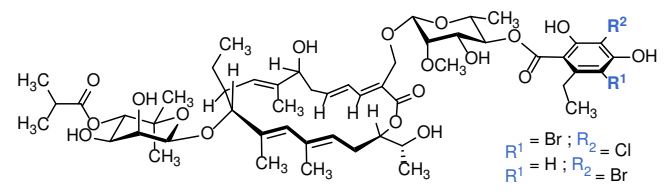

Zhang and coworkers:[44,45]

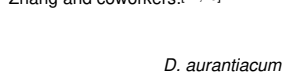
$\Delta$ tiaM mutant
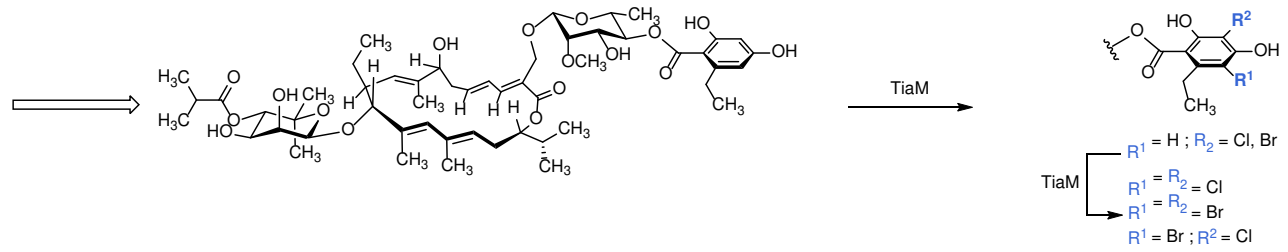

This work:
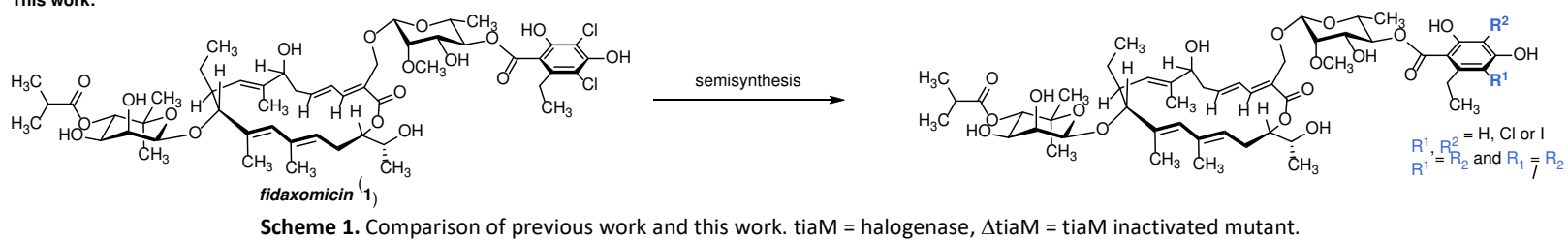

Scheme 1. Comparison of previous work and this wo
. Variations on the substituents of this aromatic ring will therefore influence its electronic properties and thereby alters the binding properties to the enzyme. Besides total synthesis studies, ${ }^{[32-39]}$ only a few examples of synthetic modifications on fidaxomicin are known to date. ${ }^{[29,40-43]}$ Researchers from Abbott Laboratories investigated the replacement of chloride by implementation of bromide into the natural product, by

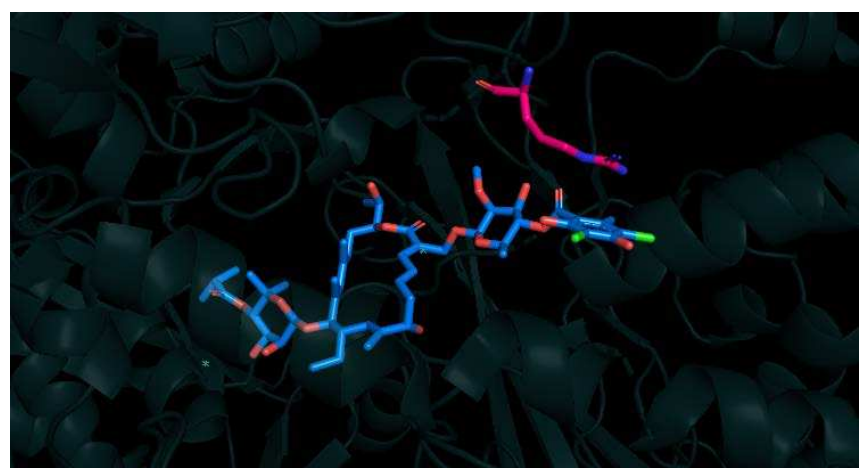

Figure 1. Section of the cryo-EM structure of fidaxomicin (1) binding to RNA polymerase (PDB-ID: 6FBV). ${ }^{[29]}$ The cation- $\pi$ interaction between $\beta^{\prime}$ R84 (magenta) and the dichloroorsellinate moiety of $\mathbf{1}$ (blue) is highlighted.

of fidaxomicin binding to RNAP offers a tool for structural design of new derivatives. Notably, these cryo-EM structures reveal a cation- $\pi$ interaction between the aromatic ring of fidaxomicin's homodichloroorsellinic acid moiety and the RNAP residue $\beta^{\prime}$ R84 (Figure 1 ). Determination of fidaxomicin's $\mathrm{pK}_{\mathrm{a}}$-value $\left(\mathrm{pK}_{\mathrm{a}}=5.6\right)^{[31]}$ confirms an acidic character of the phenol that will be mainly deprotonated in physiological environment and therefore result in a negative charge. supplying the fermentation broth of $D$. aurantiacum subsp. hamdenensis with bromide (Scheme 1). ${ }^{[44,45]}$ Later, Zhang and coworkers performed geneknockout studies to elucidate the biosynthetic pathway. ${ }^{[46,47]}$ According to their study, the halogenase TiaM is not only able to insert chloride to the homoorsellinic acid moiety, but also bromide can be introduced. However, the introduction of other halogen atoms was not achieved in this study. 
In this context, we were interested in replacing the $\mathrm{Cl}$-substituents from the natural product by semisynthetic approaches to study the influence of these substituents on the antibacterial properties of fidaxomicin.

\section{Results and Discussion}

More recent results of Zhang and coworkers also showed improved activity for dechlorinated fidaxomicin derivatives. ${ }^{[46]}$ Attracted by these results, we investigated a method for the preparation of such derivatives by using a semisynthetic approach from the natural product. Nevertheless, the direct transformation of aryl chlorides to aryl iodides is considered rather difficult. Indeed, exchange of a chlorine to a heavier halogen atom lead to the formation of a less stable compound (bond dissociation energies: $\mathrm{Ph}-\mathrm{Cl}$ (407 $\left.\mathrm{kJ} \mathrm{mol}^{-1}\right)$ vs. $\left.\mathrm{Ph}-\mathrm{I}\left(280 \mathrm{~kJ} \mathrm{~mol}^{-1}\right)\right) \cdot{ }^{[48]}$ Therefore, usually $\mathrm{Cu}-$ and $\mathrm{Ni}-$ mediated Finkelstein-type reactions are performed under harsh conditions $\left(>100^{\circ} \mathrm{C}\right)$ even for comparatively simple substrates, ${ }^{[48,49]}$ potentially limiting their synthetic application with sensitive functional groups and/or scaffolds. Nevertheless, we decided to experimentally evaluate some of these Finkelstein reactions and found that either no conversion or upon increasing the temperature to above $150{ }^{\circ} \mathrm{C}$ decomposition of $\mathbf{1}$ was observed, rendering this classical approach not suitable in our case. Introduction of electron-withdrawing substituents like acetyl-protection of the phenolic hydroxy groups was not successful neither. We therefore turned our attention to the use of milder two-step procedures involving Pd-catalyzed silylations or borylations followed by an iodination step to access the desired iodinated derivatives. ${ }^{[50,51]}$ To our delight, Buchwald's Pd-catalyzed silylation of aryl chlorides ${ }^{[51]}$ led to formation of traces of dehalogenated fidaxomicins 2, 3 and 4, which indicated that the oxidative addition step into the hindered and unreactive $\mathrm{C}-\mathrm{Cl}$ bonds of the homoorsellinic acid moiety took place.

Unfortunately, efforts to promote the reductive elimination using bulkier phosphine ligands such as $t \mathrm{Bu}$-Davephos did not lead to any improvement. Nevertheless, encouraged by this result, we thus decided to study the reactivity of $B_{2}$ pin 2 towards this reaction and we were pleased to obtain complete conversion to a mixture of mono- and didechlorinated compounds 2, 3 and $\mathbf{4}$ (Scheme 2).
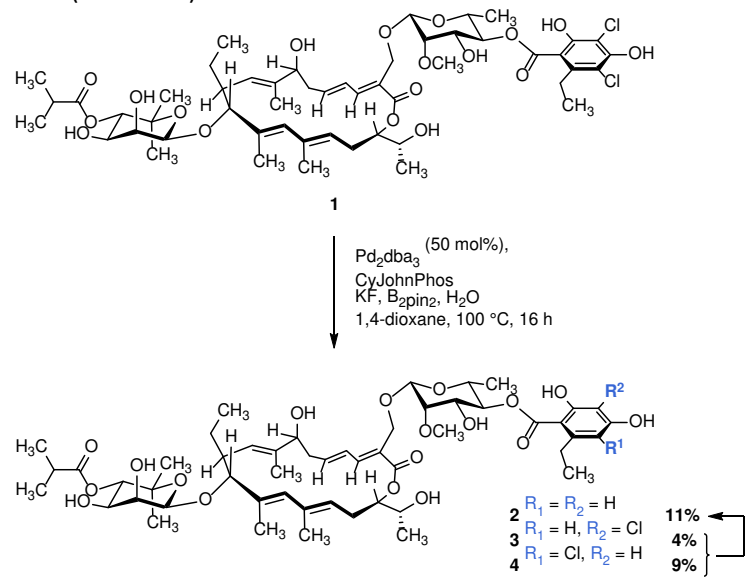

Scheme 2. Optimized conditions for the preparation of dechloro-fidaxomicin derivatives $\mathbf{2 - 4}$

Intrigued by the mechanism and the difference of reactivity between $\mathrm{Me}_{6} \mathrm{Si}_{2}$ and $\mathrm{B}_{2} \mathrm{pin}_{2}$, we performed control experiments in order to clarify the role of each reagent in these reaction conditions. Treatment of fidaxomicin under these conditions without water, base or the diboron source, respectively, led to the formation of only traces of the desired product, indicating their pivotal role.

Further mechanistic investigations using $\mathrm{D}_{2} \mathrm{O}$ led to deuterium incorporation as evidenced by UHLC-MS and NMR measurements $(40-70 \%$ Dincorporation, see $\mathrm{SI}$ ). Water as the potential $\mathrm{H}$ atom source was already reported in reductive Heck reactions and transfer hydrogenations. ${ }^{[52,53]}$ We propose that due to steric hindrance of the fully substituted aromatic system, reductive elimination is disfavored allowing formation of a Pd-hydride species which then undergoes reductive elimination to form the product (Scheme 3). ${ }^{[54]}$ Involvement of a cyclic boronate ester formed with the phenolic hydroxy group is also conceivable and the presence of many acidic, exchangeable protons in the substrate explains the moderate deuteration efficiency.

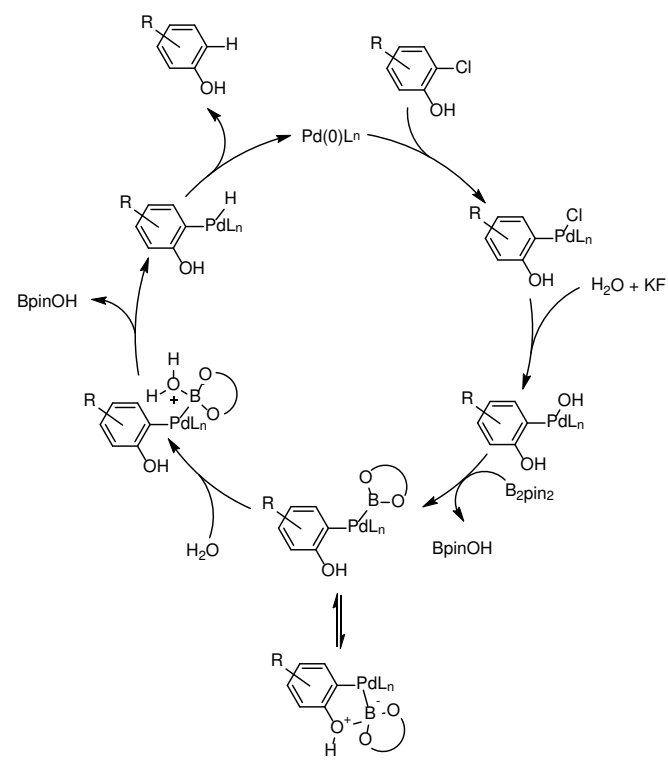

Scheme 3. Proposed mechanism for the hydrodechlorination.
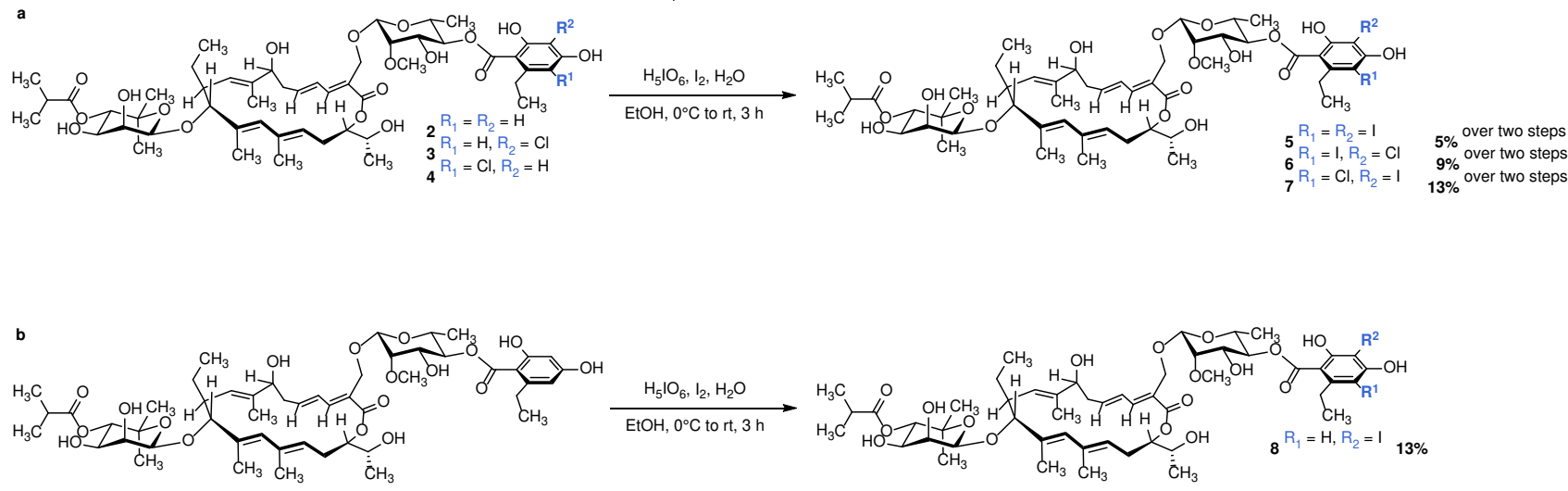

Scheme 4. lodination of dechlorinated fidaxomicins 2-4 
Having dechlorinated compounds 2-4 in hands, we further investigated the iodination via electrophilic aromatic substitution using periodic acid and iodine, ${ }^{[55,56]}$ which delivered diiodinated fidaxomicin $\mathbf{5}$ as well as monoiodinated compounds 6 and 7 (Scheme 4a). Due to the difficult purification of the dechlorination step, this reaction was performed on a crude mixture resulting in higher overall yields. Interestingly, monoiodinated compound $\mathbf{8}$ was only formed when performing the reaction on purified didechlorinated fidaxomicin 2 (Scheme 4b). We assume that residual $\mathrm{Pd}$ - or $\mathrm{B}$-impurities from the previous step might interfere with this reaction, leading to diiodofidaxomicin $\mathbf{5}$ when performing the reaction on the mixture. Additionally, chlorine to iodine exchange might also be possible under these conditions. In any case, from an analog generation perspective, these observations were useful in the context of this study.

The biological activities were evaluated by determination of the minimum inhibitory concentration (MIC) against different strains, including S. aureus, $M$. tuberculosis as well as several isolates of $C$. difficile (Figure 2 and Table 1). The dechlorinated as well as iodinated fidaxomicin analogs retain their excellent activity against $C$. difficile (Figure 2). Mono-dechlorinated compounds $\mathbf{3}$ and $\mathbf{4}$, as well as chloroiodofidaxomicin $\mathbf{6}$ and $\mathbf{7}$ display very similar activity compared to fidaxomicin (1) with only a slight decrease in activity. The same trend is evident against $S$. aureus and $M$. tuberculosis (Table

Interestingly, chloroiodofidaxomicin $\mathbf{7}$ displays improved activity against $M$. tuberculosis compared to fidaxomicin (1). Against $S$. aureus, several compounds display equal potency (e.g. $\mathbf{3}$ and $\mathbf{6}$ ) or improved antibiotic activity, such as $\mathbf{4}$ and $\mathbf{7}$.
Table 1. Minimum inhibitory concentrations $[\mu \mathrm{g} / \mathrm{mL}]$ of compounds 1-8 against $M$. tuberculosis and S. aureus

\begin{tabular}{ccc}
\hline \multirow{2}{*}{ Compound } & \multicolumn{2}{c}{ MIC $[\mathrm{\mu g} / \mathrm{mL}]$} \\
\cline { 2 - 3 } & M. tuberculosis $^{\mathrm{a}}$ & S. aureus \\
\hline $\mathbf{1}$ & 0.25 & $8-16$ \\
$\mathbf{2}$ & 0.25 & - \\
$\mathbf{3}$ & 0.25 & 8 \\
$\mathbf{4}$ & 0.5 & 4 \\
$\mathbf{5}$ & $0.25-0.5$ & 16 \\
$\mathbf{6}$ & 0.25 & 8 \\
$\mathbf{7}$ & 0.12 & $4-8$ \\
$\mathbf{8}$ & $0.25-0.5$ & 16 \\
\hline
\end{tabular}

${ }^{a}$ MIC determined on GFP-expressing M. tuberculosis strain (see Experimental Section). ${ }^{[57]}$
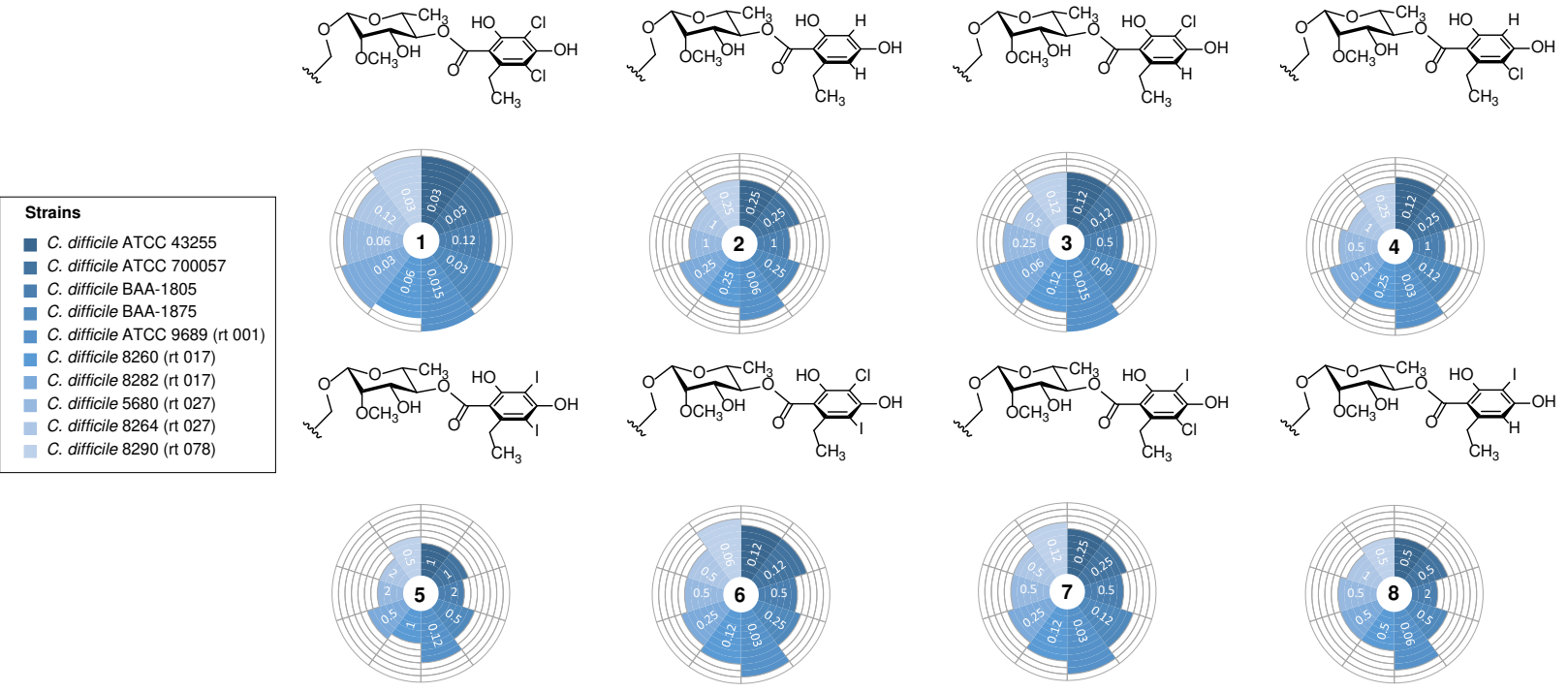

Figure 2. Minimum inhibitory concentrations $[\mu \mathrm{g} / \mathrm{mL}]$ of compounds $\mathbf{1 - 8}$ against $C$. difficile isolates.

\section{Conclusions}

In this study, we developed a semisynthetic method towards dechlorinated and iodinated fidaxomicin compounds and investigated their antibiotic activity against the Gram-positive bacterial strains $S$. aureus, $M$. tuberculosis and various isolates of $C$. difficile. These studies revealed that all derivatives maintain their excellent antibacterial properties, even though slightly decreased in comparison to the parent compound 1. Compared to already known strategies towards fidaxomicin analogs possessing substituents different from $\mathrm{Cl}$ at the aromatic moiety, this investigation represents the first semisynthetic approach towards these halogenated compounds and provides a basis for further modifications which are currently under investigation in our labs.

\section{Experimental Section} 4"',6"'-Didechlorofidaxomicin (2), 6"'-Dechlorofidaxomicin (3) and
4"',-Dechlorofidaxomicin (4)

A microwave tube was charged with commercially available fidaxomicin (1, $100 \mathrm{mg}, 94.5 \mu \mathrm{mol}, 1.0$ eq.), $\mathrm{Pd}_{2} \mathrm{dba}_{3} \cdot \mathrm{CH}_{3} \mathrm{Cl}(43.3 \mathrm{mg}, 41.8 \mu \mathrm{mol}, 44 \mathrm{~mol} \%)$, CyJohnPhos ( $49.7 \mathrm{mg}, 0.142 \mathrm{mmol}, 1.5$ eq.), KF (11.0 mg, $189 \mu \mathrm{mol}, 2.0$ eq.) and $\mathrm{B}_{2} \mathrm{pin}_{2}(72.0 \mathrm{mg}, 0.284 \mathrm{mmol}, 3.0$ eq.) and the flask was evacuated and flushed with argon several times. The solids were dissolved in dry dioxane ( $2.0 \mathrm{~mL}$, degassed by freeze-pump-thaw (3x)). Then, $\mathrm{H}_{2} \mathrm{O}(25 \mu \mathrm{L}, 1.4 \mathrm{mmol}$, 15 eq.) was added and the mixture was allowed to stir at $100^{\circ} \mathrm{C}$ for $24 \mathrm{~h}$. The reaction mixture was filtered over Celite ${ }^{\circledR}$ and the solvent was evaporated. The crude mixture was pre-purified by silica-gel column chromatography $\left(\mathrm{CH}_{2} \mathrm{Cl}_{2}\right.$ to $\mathrm{MeOH} / \mathrm{CH}_{2} \mathrm{Cl}_{2}$ 1:9) and the product containing fractions were further purified by preparative RP-HPLC (Gemini NX C18, $5 \mu$, $110 \AA, 250 \mathrm{~mm} \times 21.2 \mathrm{~mm}$; solvent $\mathrm{A}: \mathrm{H}_{2} \mathrm{O}+0.1 \% \mathrm{HCOOH}$, solvent $\mathrm{B}: \mathrm{MeCN}$ $+0.1 \% \mathrm{HCOOH} ; 20 \mathrm{~mL} / \mathrm{min}$; LC time program ( $\mathrm{min}-\% \mathrm{~B}): 0.0-50 \%, 15.0-$ $50 \%, 45.0-55 \%, 46.0-100 \%$ ] to afford $2\left(t_{R}=14.2 \mathrm{~min}, 10.2 \mathrm{mg}, 10.3 \mu \mathrm{mol}\right.$, $11 \%), 3\left(t_{R}=17.3 \mathrm{~min}, 8.7 \mathrm{mg}, 8.5 \mu \mathrm{mol}, 9 \%\right)$ and $4\left(t_{R}=18.5 \mathrm{~min}, 4.0 \mathrm{mg}\right.$, $3.9 \mu \mathrm{mol}, 4 \%)$ as colorless solids. 


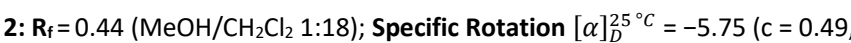
MeOH); FT-IR $\tilde{v}$ (film) 3439, 2977, 2934, 2877, 1700, 1644, 1621, 1589, 1453, $1385,1371,1320,1256,1197,1162,1070,1029,1008,901,853 \mathrm{~cm}^{-1} ;{ }^{1} \mathrm{H}$ NMR $\left(500 \mathrm{MHz}\right.$, acetone- $\left.d_{6}\right) \delta 7.23(\mathrm{~d}, J=11.4 \mathrm{~Hz}, 1 \mathrm{H}), 6.68-6.58(\mathrm{~m}, 1 \mathrm{H})$, $6.31(\mathrm{~d}, J=2.5 \mathrm{~Hz}, 1 \mathrm{H}), 6.24(\mathrm{~d}, J=2.5 \mathrm{~Hz}, 1 \mathrm{H}), 5.96$ (ddd, $J=14.7,9.6,4.6$ $\mathrm{Hz}, 1 \mathrm{H}), 5.83(\mathrm{~s}, 1 \mathrm{H}), 5.63(\mathrm{t}, J=7.0 \mathrm{~Hz}, 1 \mathrm{H}), 5.26-5.20(\mathrm{~m}, 1 \mathrm{H}), 5.12(\mathrm{t}, J=$ $9.7 \mathrm{~Hz}, 1 \mathrm{H}), 5.00(\mathrm{~d}, J=10.1 \mathrm{~Hz}, 1 \mathrm{H}), 4.78(\mathrm{~d}, J=1.2 \mathrm{~Hz}, 1 \mathrm{H}), 4.76-4.70(\mathrm{~m}$, $1 \mathrm{H}), 4.68(\mathrm{~d}, J=0.8 \mathrm{~Hz}, 1 \mathrm{H}), 4.60(\mathrm{~d}, J=11.5 \mathrm{~Hz}, 1 \mathrm{H}), 4.41(\mathrm{~d}, J=11.4 \mathrm{~Hz}, 1 \mathrm{H})$, $4.30-4.24(\mathrm{~m}, 1 \mathrm{H}), 4.09-3.94(\mathrm{~m}, 4 \mathrm{H}), 3.84-3.78(\mathrm{~m}, 2 \mathrm{H}), 3.73(\mathrm{~m}, 3 \mathrm{H})$, $3.64-3.59(\mathrm{~m}, 1 \mathrm{H}), 3.57(\mathrm{~m}, 1 \mathrm{H}), 3.51(\mathrm{~s}, 3 \mathrm{H}), 3.33-3.22(\mathrm{~m}, 1 \mathrm{H}), 2.92-$ $2.83(\mathrm{~m}, 2 \mathrm{H}), 2.81-2.62(\mathrm{~m}, 3 \mathrm{H}), 2.56(\mathrm{sept}, J=7.0 \mathrm{~Hz}, 1 \mathrm{H}), 2.52-2.40(\mathrm{~m}$, $2 \mathrm{H}), 1.98-1.90(\mathrm{~m}, 1 \mathrm{H}), 1.81(\mathrm{~d}, J=1.3 \mathrm{~Hz}, 3 \mathrm{H}), 1.73(\mathrm{~d}, J=1.3 \mathrm{~Hz}, 3 \mathrm{H}), 1.66$ $(\mathrm{s}, 3 \mathrm{H}), 1.31-1.24(\mathrm{~m}, 1 \mathrm{H}), 1.27(\mathrm{~d}, J=6.2 \mathrm{~Hz}, 3 \mathrm{H}), 1.23-1.12(\mathrm{~m}, 15 \mathrm{H}), 1.09$ $(\mathrm{s}, 3 \mathrm{H}), 0.83$ (t, $J=7.4 \mathrm{~Hz}, 3 \mathrm{H}) \mathrm{ppm} ;{ }^{13} \mathrm{C}$ NMR $\left(126 \mathrm{MHz}\right.$, acetone- $\left.d_{6}\right) \delta 176.8$, $171.7,167.8,165.8,163.4,150.6,145.4,143.4,136.9,136.12,136.09,133.8$, $128.2,126.3,125.4,124.0,111.0,105.3,101.9,101.8,96.8,93.3,81.8,78.2$ $76.7,75.7,73.8,72.9,72.8,72.4,70.8,70.2,67.7,63.4,61.7,42.0,37.3,34.8$, $30.2,28.7,28.4,26.5,20.7,19.4,19.2,18.6,18.3,17.5,16.6,15.2,13.8,11.2$ ppm; HRMS ESI(+) (MeOH) calculated for $\mathrm{C}_{52} \mathrm{H}_{76} \mathrm{O}_{18} \mathrm{Na}[\mathrm{M}+\mathrm{Na}]^{+}:$1011.49239, found: 1011.49031.

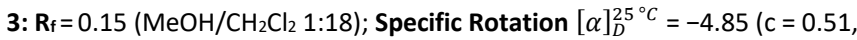
MeOH); FT-IR $\tilde{v}$ (film) 3440, 2977, 2934, 2876, 1700, 1645, 1605, 1417, 1372 $1314,1258,1198,1146,1071,1028,901,853,802,695,638,620,578,554$, $534,511,497,477,464,453 \mathrm{~cm}^{-1} ;{ }^{1} \mathrm{H}$ NMR $\left(500 \mathrm{MHz}\right.$, acetone- $\left.d_{6}\right) \delta 7.23(\mathrm{~d}$ $J=11.4 \mathrm{~Hz}, 1 \mathrm{H}), 6.63(\mathrm{dd}, J=15.0,11.5 \mathrm{~Hz}, 1 \mathrm{H}), 6.51$ (s, 1H), 5.96 (ddd, $J=$ $14.7,9.5,4.6 \mathrm{~Hz}, 1 \mathrm{H}), 5.83(\mathrm{~s}, 1 \mathrm{H}), 5.63(\mathrm{t}, J=8.3 \mathrm{~Hz}, 1 \mathrm{H}), 5.22(\mathrm{dt}, J=10.5$, $1.3 \mathrm{~Hz}, 1 \mathrm{H}), 5.13(\mathrm{t}, J=9.7 \mathrm{~Hz}, 1 \mathrm{H}), 5.00(\mathrm{~d}, J=10.1 \mathrm{~Hz}, 1 \mathrm{H}), 4.78(\mathrm{~s}, 1 \mathrm{H}), 4.76$ $-4.70(\mathrm{~m}, 1 \mathrm{H}), 4.69(\mathrm{~s}, 1 \mathrm{H}), 4.60(\mathrm{~d}, J=11.54 \mathrm{~Hz}, 1 \mathrm{H}), 4.42(\mathrm{~d}, J=11.4 \mathrm{~Hz}$, $1 \mathrm{H}), 4.29-4.24(\mathrm{~m}, 1 \mathrm{H}), 4.03$ (quint, $J=6.3 \mathrm{~Hz}, 1 \mathrm{H}), 3.95(\mathrm{~d}, J=2.3 \mathrm{~Hz}, 1 \mathrm{H}$ ), $3.82(\mathrm{dd}, J=9.8,3.4 \mathrm{~Hz}, 1 \mathrm{H}), 3.76-3.71(\mathrm{~m}, 2 \mathrm{H}), 3.65-3.60(\mathrm{~m}, 1 \mathrm{H}), 3.58$ $(\mathrm{d}, J=3.3 \mathrm{~Hz}, 1 \mathrm{H}), 3.52(\mathrm{~s}, 3 \mathrm{H}), 2.94-2.78(\mathrm{~m}, 2 \mathrm{H}), 2.77-2.60(\mathrm{~m}, 3 \mathrm{H}), 2.56$ (sept, $J=6.9 \mathrm{~Hz}, 1 \mathrm{H}), 2.51-2.38(\mathrm{~m}, 2 \mathrm{H}), 1.99-1.91(\mathrm{~m}, 1 \mathrm{H}), 1.81(\mathrm{~s}, 3 \mathrm{H})$, $1.73(\mathrm{~s}, 3 \mathrm{H}), 1.66(\mathrm{~s}, 3 \mathrm{H}), 1.30(\mathrm{~d}, J=6.1 \mathrm{~Hz}, 3 \mathrm{H}), 1.26-1.23(\mathrm{~m}, 1 \mathrm{H}), 1.23-$ $1.12(\mathrm{~m}, 15 \mathrm{H}), 1.09(\mathrm{~s}, 3 \mathrm{H}), 0.83(\mathrm{t}, J=7.4 \mathrm{~Hz}, 3 \mathrm{H}) \mathrm{ppm} ;{ }^{13} \mathrm{C}$ NMR $(126 \mathrm{MHz}$, acetone- $\left.d_{6}\right) \delta 176.8,171.5,167.8,160.8,159.1,148.0,145.4,143.4,136.9$, $136.12,136.09,133.8,128.2,126.3,125.3,124.0,110.8,106.7,106.2,101.8$, $96.8,93.3,81.8,78.2,77.3,75.7,73.8,72.86,72.81,72.3,70.7,70.2,67.7$ $63.4,61.7,42.0,37.3,34.8,29.8,28.7,28.4,26.5,20.7,19.4,19.2,18.6,18.3$ 17.5, 16.5, 15.2, 13.8, 11.2 ppm; HRMS ESI(+) (MeOH) calculated for $\mathrm{C}_{52} \mathrm{H}_{75} \mathrm{ClO}_{18} \mathrm{Na}[\mathrm{M}+\mathrm{Na}]^{+}:$1045.45341, found: 1045.45320 .

4: $\mathbf{R}_{\mathbf{f}}=0.15\left(\mathrm{MeOH} / \mathrm{CH}_{2} \mathrm{Cl}_{2}\right.$ 1:18); Specific Rotation $[\alpha]_{D}^{25^{\circ} \mathrm{C}}=-7.77$ (c = 0.95, MeOH); FT-IR $\tilde{v}$ (film) 3432, 2977, 2934, 2876, 1698, 1645, 1610, 1580, 1450, $1384,1370,1318,1243,1199,1155,1069,1028,901,848,799 \mathrm{~cm}^{-1}$; ${ }^{1}$ H NMR $\left(500 \mathrm{MHz}\right.$, acetone- $\left.d_{6}\right) \delta 7.23(\mathrm{~d}, J=11.4 \mathrm{~Hz}, 1 \mathrm{H}), 6.63(\mathrm{dd}, J=15.0,11.5 \mathrm{~Hz}$, $1 \mathrm{H}), 6.48(\mathrm{~s}, 1 \mathrm{H}), 5.96$ (ddd, $J=14.6,9.5,4.6 \mathrm{~Hz}, 1 \mathrm{H}), 5.83(\mathrm{~s}, 1 \mathrm{H}), 5.63(\mathrm{t}, J=$ $8.3 \mathrm{~Hz}, 1 \mathrm{H}), 5.22(\mathrm{dt}, J=10.5,1.3 \mathrm{~Hz}, 1 \mathrm{H}), 5.09(\mathrm{t}, J=9.7 \mathrm{~Hz}, 1 \mathrm{H}), 5.00(\mathrm{~d}, J=$ $10.1 \mathrm{~Hz}, 1 \mathrm{H}), 4.78(\mathrm{~d}, J=1.2 \mathrm{~Hz}, 1 \mathrm{H}), 4.76-4.70(\mathrm{~m}, 1 \mathrm{H}), 4.67(\mathrm{~s}, 1 \mathrm{H}), 4.60$ $(\mathrm{d}, J=11.5 \mathrm{~Hz}, 1 \mathrm{H}), 4.42(\mathrm{~d}, J=11.5 \mathrm{~Hz}, 1 \mathrm{H}), 4.29-4.24(\mathrm{~m}, 1 \mathrm{H}), 4.03$ (quint, $J=6.2 \mathrm{~Hz}, 1 \mathrm{H}), 3.96(\mathrm{~d}, J=3.0 \mathrm{~Hz}, 1 \mathrm{H}), 3.82(\mathrm{dd}, J=9.8,3.4 \mathrm{~Hz}, 1 \mathrm{H}), 3.76-$ $3.71(\mathrm{~m}, 2 \mathrm{H}), 3.65-3.60(\mathrm{~m}, 1 \mathrm{H}), 3.58(\mathrm{~d}, J=3.3 \mathrm{~Hz}, 1 \mathrm{H}), 3.52(\mathrm{~s}, 3 \mathrm{H}), 2.94-$ $2.78(\mathrm{~m}, 2 \mathrm{H}), 2.77-2.60(\mathrm{~m}, 3 \mathrm{H}), 2.56$ (sept, $J=6.9 \mathrm{~Hz}, 1 \mathrm{H}), 2.51-2.38(\mathrm{~m}$ $2 \mathrm{H}), 1.99-1.91(\mathrm{~m}, 1 \mathrm{H}), 1.81(\mathrm{~s}, 3 \mathrm{H}), 1.73(\mathrm{~s}, 3 \mathrm{H}), 1.66(\mathrm{~s}, 3 \mathrm{H}), 1.28(\mathrm{~d}, J=$ $6.2 \mathrm{~Hz}, 3 \mathrm{H}), 1.26-1.23(\mathrm{~m}, 1 \mathrm{H}), 1.23-1.12(\mathrm{~m}, 15 \mathrm{H}), 1.09(\mathrm{~s}, 3 \mathrm{H}), 0.83(\mathrm{t}, J$ $=7.4 \mathrm{~Hz}, 3 \mathrm{H}) \mathrm{ppm} ;{ }^{13} \mathrm{C}$ NMR $\left(126 \mathrm{MHz}\right.$, acetone- $\left.d_{6}\right) \delta 176.8,170.1,167.8$, $160.9,158.2,145.4,145.2,143.5,136.9,136.10,136.08,133.8,128.1,126.3$, 125.3, 124.0, 114.1, 109.5, 102.8, 101.8, 96.8, 93.3, 81.7, 78.2, 77.0, 75.7, $73.8,72.9,72.8,72.4,70.8,70.2,67.7,63.4,61.7,42.0,37.3,34.8,28.7,28.3$, 26.5, 26.3, 20.7, 19.4, 19.2, 18.6, 18.2, 17.5, 15.2, 14.4, 13.8, 11.2 ppm HRMS ESI(+) (MeOH) calculated for $\mathrm{C}_{52} \mathrm{H}_{75} \mathrm{ClO}_{18} \mathrm{Na}[\mathrm{M}+\mathrm{Na}]^{+}:$1045.45341, found: 1045.45374 .

4"',6"'-Diiodofidaxomicin (5), 6"'-lodofidaxomicin (6) and 4"', lodofidaxomicin (7)

A mixture of 2, 3 and $\mathbf{4}$ ( $94.5 \mu \mathrm{mol}, 1.0$ eq.) was dissolved in EtOH ( $1.8 \mathrm{~mL}$ ) and cooled to $0{ }^{\circ} \mathrm{C}$. Then, $I_{2}(24.0 \mathrm{mg}, 94.5 \mu \mathrm{mol}, 1.0$ eq. $)$ was added in one portion and a solution of $\mathrm{H}_{5} \mathrm{IO}_{6}(6.5 \mathrm{mg}, 28 \mu \mathrm{mol}, 0.3$ eq. $)$ in $\mathrm{H}_{2} \mathrm{O}(100 \mu \mathrm{L})$ was added dropwise. The reaction mixture was stirred at $0{ }^{\circ} \mathrm{C}$ for $1 \mathrm{~h}$ and was then allowed to slowly warm to room temperature within $2 \mathrm{~h}$. Subsequently, it was diluted with EtOAc $(10 \mathrm{~mL})$ and then quenched with $10 \%$ aq. $\mathrm{Na}_{2} \mathrm{~S}_{2} \mathrm{O}_{3}$ $(10 \mathrm{~mL})$. The phases were separated, and the aqueous layer was extracted with EtOAc $(3 \times 10 \mathrm{~mL})$. The combined organic layers were dried over $\mathrm{MgSO}_{4}$, filtered and the solvent was evaporated under reduced pressure. The crude mixture was purified by preparative RP-HPLC (Gemini NX C18, $5 \mu, 110 \AA$, $250 \mathrm{~mm} \times 21.2 \mathrm{~mm}$; solvent $\mathrm{A}: \mathrm{H}_{2} \mathrm{O}+0.1 \% \mathrm{HCOOH}$, solvent $\mathrm{B}: \mathrm{MeCN}+0.1 \%$ $\mathrm{HCOOH} ; 20 \mathrm{~mL} / \mathrm{min}$; LC time program (min -\% B): $0.0-50 \%, 15.0-50 \%$, $45.0-70 \%, 46.0-100 \%$ ] to afford, after lyophilization, $5\left(t_{R}=25.0 \mathrm{~min}, 9.5\right.$ $\mathrm{mg}, 7.6 \mu \mathrm{mol}, 9 \%), 6\left(\mathrm{t}_{\mathrm{R}}=27.5 \mathrm{~min}, 13.6 \mathrm{mg}, 11.8 \mu \mathrm{mol}, 13 \%\right)$, and 7 $\left(t_{R}=32.0 \mathrm{~min}, 6.1 \mathrm{mg}, 5.3 \mu \mathrm{mol}, 5 \%\right)$ as colorless solids.

5: $\mathbf{R}_{\mathbf{f}}=0.21\left(\mathrm{MeOH} / \mathrm{CH}_{2} \mathrm{Cl}_{2} 1: 18\right)$; Specific Rotation $[\alpha]_{D}^{24}{ }^{\circ} \mathrm{C}=-1.96$ (c = 0.26, MeOH); FT-IR $\tilde{v}$ (film) 3432, 2976, 2932, 2875, 1733, 1698, 1644, 1468, 1449, 1384, 1370, 1308, 1244, 1198, 1149, 1069, 1026, 901, 799, $767 \mathrm{~cm}^{-1} ;{ }^{1}$ H NMR $\left(500 \mathrm{MHz}\right.$, acetone- $\left.d_{6}\right) \delta 7.24(\mathrm{~d}, J=11.4 \mathrm{~Hz}, 1 \mathrm{H}), 6.68-6.58(\mathrm{~m}, 1 \mathrm{H}), 5.96$ (ddd, $J=14.7,9.6,4.6 \mathrm{~Hz}, 1 \mathrm{H}), 5.83(\mathrm{~s}, 1 \mathrm{H}), 5.63(\mathrm{t}, J=8.4 \mathrm{~Hz}, 1 \mathrm{H}), 5.22(\mathrm{~d}, J$ $=10.5 \mathrm{~Hz}, 1 \mathrm{H}), 5.10(\mathrm{t}, J=9.7 \mathrm{~Hz}, 1 \mathrm{H}), 5.00(\mathrm{~d}, J=10.1 \mathrm{~Hz}, 1 \mathrm{H}), 4.78(\mathrm{~s}, 1 \mathrm{H})$, $4.76-4.72(\mathrm{~m}, 1 \mathrm{H}), 4.68(\mathrm{~s}, 1 \mathrm{H}), 4.60(\mathrm{~d}, J=11.5 \mathrm{~Hz}, 1 \mathrm{H}), 4.43(\mathrm{~d}, J=11.5 \mathrm{~Hz}$, $1 \mathrm{H}), 4.30-4.24(\mathrm{~m}, 1 \mathrm{H}), 4.08-3.99(\mathrm{~m}, 1 \mathrm{H}), 3.95(\mathrm{~d}, J=3.5 \mathrm{~Hz}, 1 \mathrm{H}), 3.85-$ $3.78(\mathrm{~m}, 1 \mathrm{H}), 3.76-3.69(\mathrm{~m}, 2 \mathrm{H}), 3.65-3.59(\mathrm{~m}, 2 \mathrm{H}), 3.52(\mathrm{~s}, 3 \mathrm{H}), 3.13-$ $3.02(\mathrm{~m}, 2 \mathrm{H}), 2.79-2.61(\mathrm{~m}, 3 \mathrm{H}), 2.56$ (sept, $J=7.1 \mathrm{~Hz}, 1 \mathrm{H}), 2.53-2.39(\mathrm{~m}$, $2 \mathrm{H}), 1.96-1.90(\mathrm{~m}, 1 \mathrm{H}), 1.81(\mathrm{~s}, 3 \mathrm{H}),(\mathrm{m}, 15 \mathrm{H}), 1.09(\mathrm{~s}, 3 \mathrm{H}), 0.83(\mathrm{t}, \mathrm{J}=7.4$ $\mathrm{Hz}, 3 \mathrm{H}) \mathrm{ppm} ;{ }^{13} \mathrm{C}$ NMR $\left(126 \mathrm{MHz}\right.$, acetone- $\left.d_{6}\right) \delta 176.8,169.1,167.8,160.6$, $160.0,149.4,145.5,143.5,136.9,136.11,136.09,133.8,128.1,126.3,125.2$, $123.9,110.1,101.7,96.8,93.3,82.4,81.6,78.2,77.6,75.7,73.8,73.2,72.9$, $72.8,72.4,70.6,70.2,67.7,63.3,61.7,42.0,37.3,34.8,34.3,28.7,28.4,26.5$, 20.7, 19.4, 19.2, 18.6, 18.2, 17.5, 15.2, 14.7, 13.8, 11.2 ppm; HRMS ESI(+) (MeOH) calculated for $\mathrm{C}_{52} \mathrm{H}_{74} \mathrm{I}_{2} \mathrm{O}_{18} \mathrm{Na}[\mathrm{M}+\mathrm{Na}]^{+}$: 1263.28567, found: 1263.28567.

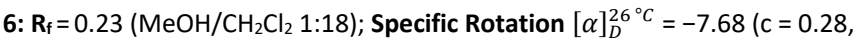
MeOH); FT-IR $\tilde{v}$ (film) 3435, 2977, 2934, 2876, 1732, 1697, 1644, 1453, 1407, 1370, 1308, 1245, 1199, 1164, 1147, 1069, 1026, 901, $799 \mathrm{~cm}^{-1} ;{ }^{1} \mathbf{H}$ NMR (500 MHz, acetone- $\left.d_{6}\right) \delta 7.24(\mathrm{~d}, J=11.5 \mathrm{~Hz}, 1 \mathrm{H}), 6.70-6.57(\mathrm{~m}, 1 \mathrm{H}), 5.96$ (ddd, $J=14.7,9.6,4.7 \mathrm{~Hz}, 1 \mathrm{H}), 5.83(\mathrm{~s}, 1 \mathrm{H}), 5.68-5.58(\mathrm{~m}, 1 \mathrm{H}), 5.22(\mathrm{~d}, J=$ $10.5 \mathrm{~Hz}, 1 \mathrm{H}), 5.09(\mathrm{t}, J=9.7 \mathrm{~Hz}, 1 \mathrm{H}), 5.00(\mathrm{~d}, J=10.1 \mathrm{~Hz}, 1 \mathrm{H}), 4.78(\mathrm{~s}, 1 \mathrm{H})$, $4.73(\mathrm{q}, J=5.3 \mathrm{~Hz}, 1 \mathrm{H}), 4.68(\mathrm{~s}, 1 \mathrm{H}), 4.60(\mathrm{~d}, J=11.5 \mathrm{~Hz}, 1 \mathrm{H}), 4.42$ (d, J = 11.5 $\mathrm{Hz}, 1 \mathrm{H}), 4.29-4.23(\mathrm{~m}, 1 \mathrm{H}), 4.08-4.00(\mathrm{~m}, 1 \mathrm{H}), 3.96(\mathrm{~d}, J=2.9 \mathrm{~Hz}, 1 \mathrm{H})$, $3.843-3.70(\mathrm{~m}, 3 \mathrm{H}), 3.64-3.57(\mathrm{~m}, 2 \mathrm{H}), 3.52(\mathrm{~s}, 3 \mathrm{H}), 3.11-3.01(\mathrm{~m}, 2 \mathrm{H})$, $2.74-2.61(\mathrm{~m}, 3 \mathrm{H}), 2.56$ (sept, $J=7.0 \mathrm{~Hz}, 1 \mathrm{H}), 2.52-2.39(\mathrm{~m}, 2 \mathrm{H}), 1.97-$ $1.87(\mathrm{~m}, 1 \mathrm{H}), 1.81(\mathrm{~s}, 3 \mathrm{H}), 1.73(\mathrm{~s}, 3 \mathrm{H}), 1.66(\mathrm{~d}, \mathrm{~J}=1.1 \mathrm{~Hz}, 3 \mathrm{H}), 1.32(\mathrm{~d}, \mathrm{~J}=6.1$ $\mathrm{Hz}, 3 \mathrm{H}), 1.30-1.23(\mathrm{~m}, 1 \mathrm{H}), 1.22-1.11(\mathrm{~m}, 15 \mathrm{H}), 1.09(\mathrm{~s}, 3 \mathrm{H}), 0.83(\mathrm{t}, \mathrm{J}=7.4$ $\mathrm{Hz}, 3 \mathrm{H}) \mathrm{ppm} ;{ }^{13} \mathrm{C}$ NMR $\left(126 \mathrm{MHz}\right.$, acetone- $\left.d_{6}\right) \delta 176.8,169.0,167.8,156.3$, $156.1,147.0,145.5,143.5,136.90,136.86,136.0,133.8,128.1,126.3,125.3$, 124.0, 111.7, 101.7, 96.8, 93.3, 82.6, 81.6, 78.2, 77.5, 75.7, 73.8, 72.8, 72.7, $72.3,70.6,70.2,67.7,63.4,61.7,42.0,37.3,34.8,33.9,28.7,28.4,26.5,20.7$, $19.4,19.2,18.6,18.2,17.5,15.2,14.8,13.8,11.2$ ppm; HRMS ESI(+) (MeOH) calculated for $\mathrm{C}_{52} \mathrm{H}_{74} \mathrm{ClIO}_{18} \mathrm{Na}[\mathrm{M}+\mathrm{Na}]^{+}:$1171.35006, found: 1171.35037 .

7: $\mathbf{R}_{\mathbf{f}}=0.23\left(\mathrm{MeOH} / \mathrm{CH}_{2} \mathrm{Cl}_{2} 1: 18\right)$; Specific Rotation $[\alpha]_{D}^{26{ }^{\circ} \mathrm{C}}=-4.28$ (c = 0.55, MeOH); FT-IR $\tilde{v}$ (film) 3433, 2977, 2934, 2876, 1732, 1697, 1644, 1453, 1385, 1370, 1310, 1242, 1198, 1163, 1148, 1069, 1026, 901, 799, $713 \mathrm{~cm}^{-1} ;{ }^{1} \mathrm{H}$ NMR $\left(500 \mathrm{MHz}\right.$, acetone- $\left.d_{6}\right) \delta 7.24(\mathrm{~d}, J=11.5 \mathrm{~Hz}, 1 \mathrm{H}), 6.68-6.58(\mathrm{~m}, 1 \mathrm{H}), 5.96$ (ddd, $J=14.6,9.5,4.6 \mathrm{~Hz}, 1 \mathrm{H}), 5.83(\mathrm{~s}, 1 \mathrm{H}), 5.65-5.60(\mathrm{~m}, 1 \mathrm{H}), 5.22(\mathrm{dt}, J=$ $10.4,1.6 \mathrm{~Hz}, 1 \mathrm{H}), 5.12(\mathrm{t}, J=9.7 \mathrm{~Hz}, 1 \mathrm{H}), 5.00(\mathrm{~d}, J=10.1 \mathrm{~Hz}, 1 \mathrm{H}), 4.78(\mathrm{~d}, J=$ $1.2 \mathrm{~Hz}, 1 \mathrm{H}), 4.76-4.71(\mathrm{~m}, 1 \mathrm{H}), 4.69(\mathrm{~s}, 1 \mathrm{H}), 4.60(\mathrm{~d}, J=11.5 \mathrm{~Hz}, 1 \mathrm{H}), 4.42$ (d, $J=11.5 \mathrm{~Hz}, 1 \mathrm{H}$ ), $4.30-4.23(\mathrm{~m}, 1 \mathrm{H}), 4.03$ (quint, $J=6.4 \mathrm{~Hz}, 1 \mathrm{H}), 3.96$ (d, $J=2.9 \mathrm{~Hz}, 1 \mathrm{H}), 3.82(\mathrm{dd}, 1 \mathrm{H}), 3.77-3.68(\mathrm{~m}, 2 \mathrm{H}), 3.67-3.54(\mathrm{~m}, 2 \mathrm{H}), 3.52$ $(\mathrm{s}, 3 \mathrm{H}), 3.11-2.96(\mathrm{~m}, 2 \mathrm{H}), 2.79-2.61(\mathrm{~m}, 3 \mathrm{H}), 2.56(\mathrm{sept}, J=78.2,6.7 \mathrm{~Hz}$, $2 \mathrm{H}), 2.52-2.39(\mathrm{~m}, 1 \mathrm{H}), 1.99-1.89(\mathrm{~m}, 1 \mathrm{H}), 1.81(\mathrm{~d}, J=1.3 \mathrm{~Hz}, 3 \mathrm{H}), 1.73(\mathrm{~d}$, $J=1.4 \mathrm{~Hz}, 3 \mathrm{H}), 1.65(\mathrm{~d}, J=1.4 \mathrm{~Hz}, 3 \mathrm{H}), 1.30(\mathrm{~d}, J=6.1 \mathrm{~Hz}, 3 \mathrm{H}), 1.28-1.25(\mathrm{~m}$, $1 \mathrm{H}), 1.25(\mathrm{t}, J=7.5 \mathrm{~Hz}, 3 \mathrm{H}), 1.20-1.11(\mathrm{~m}, 12 \mathrm{H}), 1.09(\mathrm{~s}, 3 \mathrm{H}), 0.83(\mathrm{t}, J=7.4$ $\mathrm{Hz}, 3 \mathrm{H}) \mathrm{ppm} ;{ }^{13} \mathrm{C}$ NMR $\left(126 \mathrm{MHz}\right.$, acetone- $\left.d_{6}\right) \delta 176.8,169.7,167.8,160.1$, 157.6, 145.5, 144.9, 143.5, 136.9, 136.11, 136.08, 133.8, 128.1, 126.3, 125.2, 124.0, 113.3, 109.1, 101.7, 96.8, 93.3, 81.6, 78.2, 77.8, 75.7, 73.9, 73.8, 72.9, $72.8,72.3,70.6,70.2,67.7,63.4,61.7,42.0,37.3,34.8,28.7,28.4,26.6,26.5$, 20.7, 19.4, 19.2, 18.6, 18.3, 17.5, 15.2, 14.3, 13.8, 11.1 ppm; HRMS ESI(+) $(\mathrm{MeOH})$ calculated for $\mathrm{C}_{52} \mathrm{H}_{74} \mathrm{ClIO}_{18} \mathrm{Na}[\mathrm{M}+\mathrm{Na}]^{+}$: 1171.35006, found: 1171.35027.

\section{4"'-lodo, 6"'-Dechlorofidaxomicin (8)}

Pure dechlorinated compound $\mathbf{2}$ ( $8.6 \mathrm{mg}, 8.7 \mu \mathrm{mol}, 1.0$ eq.) was dissolved in EtOH $(180 \mu \mathrm{L})$ and cooled to $0{ }^{\circ} \mathrm{C}$. Then, $\mathrm{I}_{2}(2.7 \mathrm{mg}, 10.6 \mu \mathrm{mol}, 1.2$ eq.) was added in one portion and a solution of $\mathrm{H}_{5} \mathrm{IO}_{6}(1.2 \mathrm{mg}, 5.3 \mu \mathrm{mol}, 0.6$ eq.) in $\mathrm{H}_{2} \mathrm{O}(10 \mu \mathrm{L})$ was added dropwise. The reaction mixture was allowed to stir at $0{ }^{\circ} \mathrm{C}$ for $1 \mathrm{~h}$, was diluted with EtOAc $(1 \mathrm{~mL})$ and then quenched with $10 \%$ aq. $\mathrm{Na}_{2} \mathrm{~S}_{2} \mathrm{O}_{3}(1 \mathrm{~mL})$. The phases were separated, and the aqueous layer was extracted with EtOAc $(3 \times 2 \mathrm{~mL})$. The combined organic layers were dried 
over $\mathrm{MgSO}_{4}$, filtered and the solvent was evaporated under reduced pressure. The crude mixture was purified by preparative RP-HPLC (Gemini $\mathrm{NX} \mathrm{C18,} 5 \mu, 110 \AA, 250 \mathrm{~mm} \times 21.2 \mathrm{~mm}$; solvent $\mathrm{A}: \mathrm{H}_{2} \mathrm{O}+0.1 \% \mathrm{HCOOH}$ solvent $\mathrm{B}$ : MeCN $+0.1 \% \mathrm{HCOOH} ; 20 \mathrm{~mL} / \mathrm{min}$; LC time program (min -\% B): $0.0-50 \%, 15.0-50 \%, 45.0-70 \%, 46.0-100 \%$ ] to afford, afte lyophilization, $8\left(t_{R}=22.0 \mathrm{~min}, 1.3 \mathrm{mg}, 1.2 \mu \mathrm{mol}, 13 \%\right)$ as a colorless solid.

Specific Rotation $[\alpha]_{D}^{26{ }^{\circ} \mathrm{C}}=-0.52$ (c=0.58, MeOH); FT-IR $\tilde{v}$ (film) 3441, 2977 2933, 2876, 1697, 1643, 1598, 1455, 1407, 1385, 1371, 1311, 1255, 1199, $1163,1147,1116,1069,1029,901,846,802,772,716,629,573,530,499$, 474, $464 \mathrm{~cm}^{-1} ;{ }^{1} \mathrm{H}$ NMR (500 MHz, acetone- $\left.d_{6}\right) \delta 7.23(\mathrm{~d}, J=11.4 \mathrm{~Hz}, 1 \mathrm{H})$, $6.69-6.56(\mathrm{~m}, 1 \mathrm{H}), 6.51(\mathrm{~s}, 1 \mathrm{H}), 5.96(\mathrm{~s}, 1 \mathrm{H}), 5.88-5.78(\mathrm{~m}, 1 \mathrm{H}), 5.63(\mathrm{t}, J=$ $8.3 \mathrm{~Hz}, 1 \mathrm{H}), 5.22(\mathrm{dt}, J=10.5,1.5 \mathrm{~Hz}, 1 \mathrm{H}), 5.14(\mathrm{t}, J=9.7 \mathrm{~Hz}, 1 \mathrm{H}), 5.00(\mathrm{~d}, J=$ $10.1 \mathrm{~Hz}, 1 \mathrm{H}), 4.78(\mathrm{~d}, J=1.4 \mathrm{~Hz}, 1 \mathrm{H}), 4.73(\mathrm{q}, J=5.3 \mathrm{~Hz}, 1 \mathrm{H}), 4.69(\mathrm{~d}, J=0.9$ $\mathrm{Hz}, 1 \mathrm{H}), 4.60(\mathrm{~d}, J=11.4 \mathrm{~Hz}, 1 \mathrm{H}), 4.42(\mathrm{~d}, J=11.5 \mathrm{~Hz}, 1 \mathrm{H}), 4.29-4.25(\mathrm{~m}$, $1 \mathrm{H}), 4.07-4.00(\mathrm{~m}, 1 \mathrm{H}), 3.96(\mathrm{~s}, 1 \mathrm{H}), 3.85-3.80(\mathrm{~m}, 1 \mathrm{H}), 3.77-3.71(\mathrm{~m}$, $2 \mathrm{H}), 3.63(\mathrm{dd}, J=9.6,6.2 \mathrm{~Hz}, 1 \mathrm{H}), 3.58(\mathrm{~d}, J=3.2 \mathrm{~Hz}, 1 \mathrm{H}), 3.52(\mathrm{~s}, 3 \mathrm{H}), 2.92-$ $2.84(\mathrm{~m}, 2 \mathrm{H}), 2.77-2.61(\mathrm{~m}, 3 \mathrm{H}), 2.56(\mathrm{sept}, J=6.8 \mathrm{~Hz}, 1 \mathrm{H}), 2.55-2.39(\mathrm{~m}$, $2 \mathrm{H}), 1.99-1.90(\mathrm{~m}, 1 \mathrm{H}), 1.81(\mathrm{~d}, J=1.4 \mathrm{~Hz}, 3 \mathrm{H}), 1.73(\mathrm{~d}, J=1.4 \mathrm{~Hz}, 3 \mathrm{H}), 1.66$ $(\mathrm{s}, 3 \mathrm{H}), 1.28(\mathrm{~d}, J=6.2 \mathrm{~Hz}, 3 \mathrm{H}), 1.24(\mathrm{~d}, 1 \mathrm{H}), 1.21(\mathrm{t}, J=7.4 \mathrm{~Hz}, 3 \mathrm{H}), 1.19-$ $1.12(\mathrm{~m}, 12 \mathrm{H}), 1.09(\mathrm{~s}, 3 \mathrm{H}), 0.83(\mathrm{t}, J=7.4 \mathrm{~Hz}, 3 \mathrm{H}) \mathrm{ppm} ;{ }^{13} \mathrm{C}$ NMR $(126 \mathrm{MHz}$, acetone- $\left.d_{6}\right) \delta 176.8,171.5,167.8,164.5,163.4,150.3,145.4,143.4,136.9$, $136.12,136.09,133.8,128.2,126.3,125.3,124.0,110.3,105.2,101.8,96.8$, $93.1,81.8,78.2,77.3,75.7,73.8,72.84,72.80,72.75,72.3,70.8,70.1,67.7$ $63.4,61.7,42.0,37.3,34.8,30.0,28.7,28.4,26.5,20.7,19.4,19.2,18.6,18.3$ 17.5, 16.5, 15.2, 13.8, $11.2 \mathrm{ppm}$; HRMS ESI(+) (MeOH) calculated for $\mathrm{C}_{52} \mathrm{H}_{75} \mathrm{IO}_{18} \mathrm{Na}[\mathrm{M}+\mathrm{Na}]^{+}:$1137.38903, found: 1137.38932 .

\section{General Procedure for the determination of MIC values for S. aureus}

The strain Staphylococcus aureus ATCC 29213 was grown overnight at $37^{\circ} \mathrm{C}$ on $\mathrm{MH}$ II agar plates. (BD ${ }^{\mathrm{TM}} \mathrm{BBL}^{\mathrm{TM}}$ Mueller Hinton II Agar, BD Diagnostics). MIC values were determined by broth dilution method according to the recommendations of the Clinical and Laboratory Standards Institute (CSLI; U.S.A.). The inoculum size was about $7.5 \times 10^{5}$ colony forming units/well. The compounds were diluted in $\mathrm{H}_{2} \mathrm{O}$ from $1.0 \mathrm{mg} / \mathrm{mL}$ stock solutions in $50 \%$ methanol/ $\mathrm{H}_{2} \mathrm{O}$ in a 2 -fold dilution series. The microtiter plates were incubated at $37{ }^{\circ} \mathrm{C}$ overnight. Afterwards, the MIC (lowest concentration of the compounds with no bacterial growth observed) was determined by visual inspection.

\section{General procedure for the determination of MIC values for $M$. tuberculosis}

MIC determination was essentially conducted as described recently. ${ }^{[57]}$ Briefly, the Green-Fluorescent Protein (GFP) expressing recombinant Mycobacterium tuberculosis H37Rv $\mathrm{rpsL}^{[58]}$ transformed with pOLYG-PrGFP $^{[59]}$ was grown in Middlebrook 7H9-OADC with $0.05 \%$ Tween 80 until mid-log phase (optical density at $600 \mathrm{~nm}$ OD600 $=0.3-1.0$ ), diluted to an OD600 of 0.04 and $20 \mu \mathrm{l}$ of the suspension were added to an equal volume of 12-point two-fold serial dilutions of the compounds in 7H9-OADC-Tween in 384-well plates in triplicates. Compound concentrations were in the range of 62.5 to $0.031 \mu \mathrm{M}$. Fluorescence was measured immediately after inoculation (background) and after 10 days of incubation at $37{ }^{\circ} \mathrm{C}$. Dose response curves were fitted with a 4-parameter log-normal model. PMIN $[-,-]$ and PMAX $[-, 120]$ are the minimum and the maximum, respectively, PHill [0,-] indicates the steepness, and EC50 [-,-] the log-back transformed Minimal Effective Concentration 50. The computational and statistical analysis was conducted with R (3.0.1 - 3.1.1; https://www.r-project.org/). Dose response curves were fitted with the 'drc' package. The inhibitory potency I was calculated with the equation I $=100-[100 \bullet(S-P) / N-P)] . S$ is the sample's fluorescence while $\mathrm{P}$ and $\mathrm{N}$ derive from growth inhibition with the control drug (Kanamycin A) and solvent growth control measurements (DMSO $1.25 \%$ vol./vol.), respectively. A fluorescence reduction of $90 \%$ as compared to the no-drug control was reported as Minimal Inhibitory Concentration (MIC90).

\section{General procedure for the determination of MIC values for $C$. difficile}

MIC determination was carried out by Micromyx, LLC, 4717 Campus Drive, Kalamazoo, MI, USA 49008.

Approximately $5 \mathrm{mg}$ of each of the test compounds were provided. These were stored at $-20^{\circ} \mathrm{C}$ until testing. On the day of the assay, the test articles were dissolved in $100 \%$ DMSO (dimethyl sulfoxide, Sigma; St. Louis, MO, Cat. No. 472301-500ML, Lot No. SHBH5551V) to a stock concentration of 3232 $\mu \mathrm{g} / \mathrm{mL}$. The concentration range tested for these test agents was $16-0.015$ $\mu \mathrm{g} / \mathrm{mL}$. The comparator agents, metronidazole and clindamycin were supplied by Micromyx, as shown in the table below:

\begin{tabular}{llllll}
\hline $\begin{array}{l}\text { Compar- } \\
\text { ator } \\
\text { Drug }\end{array}$ & Supplier & $\begin{array}{l}\text { Catalog } \\
\text { No. }\end{array}$ & Lot No. & $\begin{array}{l}\text { Solvent/Dil } \\
\text { uent }\end{array}$ & $\begin{array}{l}\text { Testing } \\
\text { Range } \\
(\mu \mathrm{g} / \mathrm{mL})\end{array}$ \\
\hline $\begin{array}{l}\text { Metro- } \\
\text { nidazole }\end{array}$ & Sigma & $\begin{array}{l}\text { M3761- } \\
100 \mathrm{G}\end{array}$ & $\begin{array}{l}095 \mathrm{~K} 069 \\
\mathrm{DMSO} /\end{array}$ & $\begin{array}{l}\mathrm{DH} \\
\mathrm{dH2O}\end{array}$ & $64-0.06$ \\
$\begin{array}{l}\text { Clinda- } \\
\text { mycin }\end{array}$ & Sigma & $\begin{array}{l}\text { C5269- } \\
100 \mathrm{MG}\end{array}$ & $021 \mathrm{M} 153$ & $\begin{array}{l}\mathrm{dH2O} / \\
\mathrm{dH2O}\end{array}$ & $32-0.03$ \\
\hline
\end{tabular}

\section{Test Organisms}

Test organisms consisted of reference strains from the American Type Culture Collection (ATCC; Manassas, VA) and clinical isolates from the Micromyx repository (MMX; Kalamazoo, MI). Organisms were initially received at Micromyx and were streaked for isolation. Colonies were picked by sterile swab from the medium and suspended in the appropriate broth containing cryoprotectant. The suspensions were aliquoted into cryogenic vials and maintained at $-80^{\circ} \mathrm{C}$.

Prior to testing, all isolates were streaked onto Brucella Agar supplemented with hemin, Vitamin K and 5\% sheep blood (Becton Dickinson [BD]; Sparks, MD, Cat. No. 297716, Lot No. 8256909) and incubated anaerobically at 35$37^{\circ} \mathrm{C}$ for $44-48$ hours.

Additionally, Bacteroides fragilis ATCC 25285 and Clostridium difficile ATCC 700057 were tested for purposes of quality control.

\section{Test Medium}

The medium employed for anaerobic testing in the broth microdilution MIC assay was Brucella Broth (BD, Cat. No. 211088, Lot No. 7128995), supplemented with hemin (Sigma, Lot No. SLBP5720V), Vitamin K (Sigma, Lot No. MKCG2075) and 5\% laked horse blood (LHB, Cleveland Scientific; Bath, OH, Lot No. 474990).

\section{Broth Microdilution Assay}

The MIC assay method followed the procedure described by the CLSI[60,61] and employed automated liquid handlers (Multidrop 384, Labsystems, Helsinki, Finland; Biomek 2000 and Biomek FX, Beckman Coulter, Fullerton CA) to conduct serial dilutions and liquid transfers. The wells in columns 2 through 12 in a standard 96-well microdilution plate (Costar) were filled with $150 \mu \mathrm{L}$ of the appropriate diluent (DMSO for the test agents; dH2O for metronidazole and clindamycin). The drugs ( $300 \mu \mathrm{L}$ at $101 \mathrm{X}$ the desired top concentration in the test plates) were dispensed into the appropriate well in column 1 of the mother plates. The Biomek 2000 was used to make serial 2 -fold dilutions through column 11 in the "mother plate". The wells of column 12 contained no drug and were the organism growth control wells.

The daughter plates for testing of all isolates were loaded with $190 \mu \mathrm{L}$ per well of supplemented Brucella broth with 5\% LHB using the Multidrop 384. The daughter plates were prepared on the Biomek FX instrument which transferred $2 \mu \mathrm{L}$ of $101 \mathrm{X}$ drug solution from each well of a mother plate to the corresponding well of each daughter plate in a single step. The wells of the daughter plates ultimately contained $190 \mu \mathrm{L}$ of medium, $2 \mu \mathrm{L}$ of drug solution, and $10 \mu \mathrm{L}$ of bacterial inoculum prepared in broth.

A standardized inoculum of each organism was prepared per CLSI methods. ${ }^{[60,61]}$ For all bacteria, suspensions were prepared in supplemented Brucella broth supplemented with hemin and Vitamin $K$ to equal the turbidity of a $0.5 \mathrm{McF}$ arland standard. These suspensions were further diluted 1:10 in supplemented Brucella broth with 5\% LHB. The inoculum was dispensed into sterile reservoirs (Beckman Coulter) and transferred by hand in the Bactron Anaerobe chamber so that inoculation took place from low to high drug concentration. A $10 \mu \mathrm{L}$ aliquot of inoculum was delivered into each well. Inoculated daughter plates were stacked and placed in an anaerobic box with GasPak sachets (BD; Lot No. 6309689), covered with a lid on the top plate, and incubated at $35-37^{\circ} \mathrm{C}$.

The microplates were viewed from the bottom using a plate viewer after 46 hours. For each mother plate, an un-inoculated solubility control plate was 
observed for evidence of drug precipitation. The MIC was read and recorded as the lowest concentration of drug that inhibited visible growth of the organism.

\section{Supplementary Material}

The NMR spectra are available in the Supporting Information.

\section{Acknowledgements}

We thank the Swiss National Science Foundation for financial support (182043 (K.G.) and 31003A_153349/1 (P.S.)). Work in the laboratory of P.S. is supported by Swiss Lung Association (2018-02). We gratefully acknowledge the NMR- und MS services of the University of Zurich determination and D. Dailler for proofreading the manuscript.

\section{Author Contribution Statement}

A. D. and K. G. designed the study. A. D. carried out the synthesis and characterization of the derivatives. A. D. and K. G. analyzed and discussed the results. I. S. S. determined MIC values against S. aureus. D. S. and P. S. developed the MIC tests for $M$. tuberculosis and performed the biological evaluation of the derivatives. A. D. and K. G. wrote the manuscript.

A patent application (WO2019135010A1, EP18150671.8A) was filed Jan $8^{\text {th }}$, 2018 that includes antibiotics presented in this work.

\section{References}

[1] F. Parenti, H. Pagani, G. Beretta, 'Lipiarmycin, A New Antibiotic From Actinoplanes I. Description of the Producer Strain and Fermentation Studies', J. Antibiot. 1975, 28, 247-252.

[2] C. Coronelli, R. J. White, G. C. Lancini, F. Parenti, 'Lipiarmycin, A New Antibiotic from Actinoplanes II. Isolation, Chemical, Biological and Biochemical Charaterization', J. Antibiot. 1975, 28, 253-259.

[3] C. Coronelli, F. Parenti, R. White, H. Pagani, Lipiarmycin and Its Preparation, 1976, US 3,978,211.

[4] S. Õmura, N. Imamura, R. Õiwa, H. Kuga, R. Iwata, R. Masuma, Y. Iwai, 'Clostomicins, New Antibiotics Produced by Micromonospora Echinospora subsp. Armeniaca subsp. nov. I. Production, Isolation, and Physico-Chemical and Biological Properties', J. Antibiot. 1986, 39, 1407-1412.

[5] Y. Takashi, Y. Iwai, S. Õmura, 'Clostomicins, New Antibiotics Produced by Micromonospora Echinospora subsp. Armeniaca subsp. nov. II. Taxonomic Study of the Producing Microorganism', J. Antibiot. 1986, 39, 1413-1418.

[6] R. J. Theriault, J. P. Karwowski, M. Jackson, R. L. Girolami, G. N. Sunga, C. M. Vojtko, L. J. Coen, 'Tiacumicins, a Novel Complex of 18-Membered Macrolide Antibiotics I. Taxonomy, Fermentation and Antibacterial Activity', J. Antibiot. 1987, 40, 567-574.

[7] J. E. Hochlowski, S. J. Swanson, L. M. Ranfranz, D. N. Whittern, A. M. Buko, J. B. McAlpine, 'Tiacumicins, a Novel Complex of 18Membered Macrolides II. Isolation and Structure Determination', J. Antibiot. 1987, 40, 575-588.

[8] M. Kurabachew, S. H. J. Lu, P. Krastel, E. K. Schmitt, B. L. Suresh, A. Goh, J. E. Knox, N. L. Ma, J. Jiricek, D. Beer, M. Cynamon, F. Petersen, V. Dartois, T. Keller, T. Dick, V. K. Sambandamurthy, 'Lipiarmycin targets RNA polymerase and has good activity against multidrug-resistant strains of Mycobacterium tuberculosis', J. Antimicrob. Chemother. 2008, 62, 713-719.

[9] W. Erb, J. Zhu, 'From natural product to marketed drug: the tiacumicin odyssey', Nat. Prod. Rep. 2013, 30, 161-174.

[10] A. Dorst, K. Gademann, 'Chemistry and Biology of the Clinically Used Macrolactone Antibiotic Fidaxomicin', Helv. Chim. Acta 2020, 103, e2000038.

[11] A. Dorst, E. Jung, K. Gademann, 'Recent Advances in Mode of Action and Biosynthesis Studies of the Clinically Used Antibiotic
Fidaxomicin', Chimia 2020, 74, 270-273.

H. Mikamo, K. Tateda, K. Yanagihara, S. Kusachi, Y. Takesue, T. Miki, Y. Oizumi, K. Gamo, A. Hashimoto, J. Toyoshima, K. Kato, 'Efficacy and safety of fidaxomicin for the treatment of Clostridioides (Clostridium) difficile infection in a randomized, double-blind, comparative Phase III study in Japan', J. Infect. Chemother. 2018, 24, 744-752.

[13] R. N. Swanson, D. J. Hardy, N. L. Shipkowitz, C. W. Hanson, N. C. Ramer, P. B. Fernandes, J. J. Clement, 'In Vitro and In Vivo Evaluation of Tiacumicins $\mathrm{B}$ and $\mathrm{C}$ against Clostridium difficile', Antimicrob. Agents Chemother. 1991, 35, 1108-1111.

[14] Y. K. Shue, P. S. Sears, S. Shangle, R. B. Walsh, C. Lee, S. L. Gorbach, F. Okumu, R. A. Preston, 'Safety, Tolerance, and Pharmacokinetic Studies of OPT-80 in Healthy Volunteers following Single and Multiple Oral Doses', Antimicrob. Agents Chemother. 2008, 52, 1391-1395.

[15] Z. Peng, D. Jin, H. B. Kim, C. W. Stratton, B. Wu, Y.-W. Tang, X. Sun, 'Update on antimicrobial resistance in Clostridium difficile: Resistance mechanisms and antimicrobial susceptibility testing', J. Clin. Microbiol. 2017, 55, 1998-2008.

[16] World Health Organization, Global Action Plan on Antimicrobial Resistance, 2015.

[17] Bundesamt für Gesundheit, Strategie Antibiotikaresistenzen Schweiz, 2015.

[18] F. E. Koehn, 'Biosynthetic medicinal chemistry of natural product drugs', MedChem Comm 2012, 3, 854-865.

[19] D. J. Newman, G. M. Cragg, 'Natural Products as Sources of New Drugs from 1981 to 2014 ', J. Nat. Prod. 2016, 79, 629-661.

[20] P. M. Wright, I. B. Seiple, A. G. Myers, 'The evolving role of chemical synthesis in antibacterial drug discovery', Angew. Chem. Int. Ed. 2014, 53, 8840-8869.

[21] I. B. Seiple, Z. Zhang, P. Jakubec, A. Langlois-Mercier, P. M. Wright, D. T. Hog, K. Yabu, S. R. Allu, T. Fukuzaki, P. N. Carlsen, Y. Kitamura, X. Zhou, M. L. Condakes, F. T. Szczypiski, W. D. Green, A. G. Myers, 'A platform for the discovery of new macrolide antibiotics', Nature 2016, 533, 338-345.

[22] M. Talpaert, F. Campagnari, L. Clerici, 'Lipiarmycin: An Antibiotic Inhibiting Nucleic Acid Polymerases', Biochem. Biophys. Res. Commun. 1975, 63, 328-334.

[23] A. L. Sonenshein, H. B. Alexander, D. M. Rothstein, S. H. Fisher, 'Lipiarmycin-Resistant Ribonucleic Acid Polymerase Mutants of Bacillus subtilis.', J. Bacteriol. 1977, 132, 73-79.

[24] A. L. Sonenshein, H. B. Alexander, 'Initiation of Transcription in vitro is Inhibited by Lipiarmycin', J. Mol. Biol. 1979, 127, 55-72. A. Tupin, M. Gualtieri, J. P. Leonetti, K. Brodolin, 'The transcription inhibitor lipiarmycin blocks DNA fitting into the RNA polymerase catalytic site', EMBO J. 2010, 29, 2527-2537.

[26] A. Srivastava, M. Talaue, S. Liu, D. Degen, R. Y. Ebright, E. Sineva, A. Chakraborty, S. Y. Druzhinin, S. Chatterjee, J. Mukhopadhyay, Y. W. Ebright, A. Zozula, J. Shen, S. Sengupta, R. R. Niedfeldt, C. Xin, T. Kaneko, H. Irschik, R. Jansen, S. Donadio, N. Connell, R. H. Ebright, 'New target for inhibition of bacterial RNA polymerase: "switch region"', Curr. Opin. Microbiol. 2011, 14, 532-543.

[27] I. Artsimovitch, J. Seddon, P. Sears, 'Fidaxomicin Is an Inhibitor of the Initiation of Bacterial RNA Synthesis', Clin. Infect. Dis. 2012, 55, 127-131.

[28] Z. Morichaud, L. Chaloin, K. Brodolin, 'Regions 1.2 and 3.2 of the RNA Polymerase $\sigma$ Subunit Promote DNA Melting and Attenuate Action of the Antibiotic Lipiarmycin', J. Mol. Biol. 2016, 428, 463476.

[29] W. Lin, K. Das, D. Degen, C. Zhang, R. H. Ebright, W. Lin, K. Das, D. Degen, A. Mazumder, D. Duchi, D. Wang, Y. W. Ebright, 'Structural Basis of Transcription Inhibition by Fidaxomicin (Lipiarmycin A3)', Mol. Cell 2018, 70, 60-71.

[30] H. Boyaci, J. Chen, M. Lilic, M. Palka, R. A. Mooney, R. Landick, S. A. Darst, E. A. Campbell, 'Fidaxomicin jams Mycobacterium tuberculosis RNA polymerase motions needed for initiation via RbpA contacts', Elife 2018, 7, 1-19.

[31] H. Hattori, E. Kaufmann, H. Miyatake-Ondozabal, R. Berg, K. Gademann, 'Total Synthesis of Tiacumicin A. Total Synthesis, Relay Synthesis, and Degradation Studies of Fidaxomicin (Tiacumicin B, Lipiarmycin A3)', J. Org. Chem. 2018, 83, 71807205. 
[32] H. Miyatake-Ondozabal, E. Kaufmann, K. Gademann, 'Total Synthesis of the Protected Aglycon of Fidaxomicin (tiacumicin B, Lipiarmycin A3)', Angew. Chem. Int. Ed. 2015, 54, 1933-1936.

[33] W. Erb, J. M. Grassot, D. Linder, L. Neuville, J. Zhu 'Enantioselective Synthesis of Putative Lipiarmycin Aglycon Related to Fidaxomicin/Tiacumicin B', Angew. Chem. Int. Ed. 2015, 54, 1929-1932.

[34] F. Glaus, K. H. Altmann, 'Total Synthesis of the Tiacumicin B (Lipiarmycin A3/Fidaxomicin) Aglycone', Angew. Chem. Int. Ed. 2015, 54, 1937-1940.

[35] E. Kaufmann, H. Hattori, H. Miyatake-Ondozabal, K. Gademann, 'Total Synthesis of the Glycosylated Macrolide Antibiotic Fidaxomicin', Org. Lett. 2015, 17, 3514-3517.

[36] L. Jeanne-Julien, G. Masson, E. Astier, G. Genta-Jouve, V. Servajean, J. M. Beau, S. Norsikian, E. Roulland, 'Synthesis of a Tiacumicin B Protected Aglycone', Org. Lett. 2017, 19, 40064009.

[37] E. Roulland, 'Tiacumicin B: An Antibiotic of Prime Importance and a Natural Product with an Inspiring Complex Structure', Synthesis 2018, 50, 4189-4200.

[38] L. Jeanne-Julien, G. Masson, E. Astier, G. Genta-Jouve, V. Servajean, J.-M. Beau, S. Norsikian, E. Roulland, 'Study of the Construction of the Tiacumicin B Aglycone', J. Org. Chem. 2018, 83, 921-929.

[39] S. Norsikian, C. Tresse, M. François-Eude, L. Jeanne-Julien, G. Masson, V. Servajean, G. Genta-Jouve, J.-M. Beau, E. Roulland, 'Total Synthesis of Tiacumicin B: Implementing H-bond-Directed Acceptor Delivery for Highly Selective $\beta$-Glycosylations', Angew. Chem. Int. Ed. 2020, 59, 6612-6616.

[40] M.-C. Wu, C.-C. Huang, Y.-C. Lu, W.-J. Fan, Derivatives of Tiacumicin B as Anti-Cancer Agents, 2008, US 2009/0110718 A1.

[41] J. E. McAlpine, J. E. Hochlowski, Dialkyltiacumicin Compounds, 1996, US 5,583,115.

[42] D. Dailler, A. Dorst, D. Schäfle, P. Sander, K. Gademann, 'Novel Fidaxomicin Antibiotics through Site-Selective Catalysis', ChemRxiv 2020, DOI: 10.26434/chemrxiv.12593429.v1.

[43] A. Dorst, R. Berg, C. G. W. Gertzen, D. Schäfle, K. Zerbe, M. Gwerder, S. D. Schnell, P. Sander, H. Gohlke, K. Gade-, 'Semisynthetic Analogs of the Antibiotic Fidaxomicin - Design, Synthesis, and Biological Evaluation', ChemRxiv 2020, DOI: 10.26434/chemrxiv.12613823.v1.

[44] J. E. Hochlowski, M. Jackson, R. R. Rasmussen, A. M. Buko, J. J. Clement, D. N. Whittern, J. B. McAlpine, 'Production of Brominated Tiacumicin Derivatives', J. Antibiot. 1997, 50, 201205.

[45] J. E. Hochlowski, M. Jackson, J. B. McAlpine, R. R. Rasmussen, Bromotiacumicin Compounds, 1998, US 5,767,096.

[46] H. Zhang, X. Tian, X. Pu, Q. Zhang, W. Zhang, C. Zhang, 'Tiacumicin Congeners with Improved Antibacterial Activity from a Halogenase-Inactivated Mutant', J. Nat. Prod. 2018, 81, 12191224.

[47] Y. Xiao, S. Li, S. Niu, L. Ma, G. Zhang, H. Zhang, G. Zhang, J. Ju, C. Zhang, 'Characterization of Tiacumicin B Biosynthetic Gene Cluster Affording Diversified Tiacumicin Analogues and Revealing a Tailoring Dihalogenase', J. Am. Chem. Soc. 2011, 133, 10921105.

[48] T. D. Sheppard, 'Metal-catalysed halogen exchange reactions of aryl halides', Org. Biomol. Chem. 2009, 7, 1043-1052.

[49] G. Evano, A. Nitelet, P. Thilmany, D. F. Dewez, 'Metal-Mediated Halogen Exchange in Aryl and Vinyl Halides: A Review', Front. Chem. 2018, 6, 1-18.

[50] C. Thiebes, G. K. S. Prakash, N. A. Petasis, G. A. Olah, 'Mild Preparation of Haloarenes by Ipso-Substitution of Arylboronic Acids with N-Halosuccinimides', Synlett 1998, 141-142.

[51] E. McNeill, T. E. Barder, S. L. Buchwald, 'Palladium-catalyzed silylation of aryl chlorides with hexamethyldisilane', Org. Lett. 2007, 9, 3785-3788.

[52] W. Kong, Q. Wang, J. Zhu, 'Water as a Hydride Source in Palladium-Catalyzed Enantioselective Reductive Heck Reactions', Angew. Chem. Int. Ed. 2017, 56, 3987-3991.

[53] S. P. Cummings, T. N. Le, G. E. Fernandez, L. G. Quiambao, B. J. Stokes, 'Tetrahydroxydiboron-Mediated Palladium-Catalyzed Transfer Hydrogenation and Deuteriation of Alkenes and Alkynes
Using Water as the Stoichiometric H or D Atom Donor', J. Am. Chem. Soc. 2016, 138, 6107-6110.

[54] A. A. Thomas, S. E. Denmark, 'Pre-transmetalation intermediates in the Suzuki-Miyaura reaction revealed: The missing link', Science 2016, 352, 329-332.

[55] B. A. Hathaway, K. L. White, M. E. McGill, 'Comparison of iodination of methoxylated benzaldehydes and related compounds using iodine/silver nitrate and iodine/periodic acid', Synth. Commun. 2007, 37, 3855-3860.

[56] C. W. Am Ende, Z. Zhou, K. A. Parker, 'Total synthesis of ( \pm )bisabosqual A', J. Am. Chem. Soc. 2013, 135, 582-585.

[57] M. Dal Molin, P. Selchow, D. Schäfle, A. Tschumi, T. Ryckmans, S. Laage-Witt, P. Sander, 'Identification of novel scaffolds targeting Mycobacterium tuberculosis', J. Mol. Med. 2019, 97, 1601-1613.

[58] C. Raynaud, K. G. Papavinasasundaram, R. A. Speight, B. Springer, P. Sander, E. C. Böttger, M. J. Colston, P. Draper, 'The functions of OmpATb, a pore-forming protein of Mycobacterium tuberculosis', Mol. Microbiol. 2002, 46, 191-201.

[59] U. Matt, P. Selchow, M. Dal Molin, S. Strommer, O. Sharif, K. Schilcher, F. Andreoni, A. Stenzinger, A. S. Zinkernagel, M. Zeitlinger, P. Sander, J. Nemeth, 'Chloroquine enhances the antimycobacterial activity of isoniazid and pyrazinamide by reversing inflammation-induced macrophage efflux', Int. J. Antimicrob. Agents 2017, 50, 55-62.

[60] Clinical and Laboratory Standards Institute (CLSI), Methods for Antimicrobial Susceptibility Testing of Anaerobic Bacteria: Ninth Edition, 2018.

[61] Clinical and Laboratory Standards Institute (CLSI), Performance Standards for Antimicrobial Susceptibility Testing: 29th Edition, 2019. 


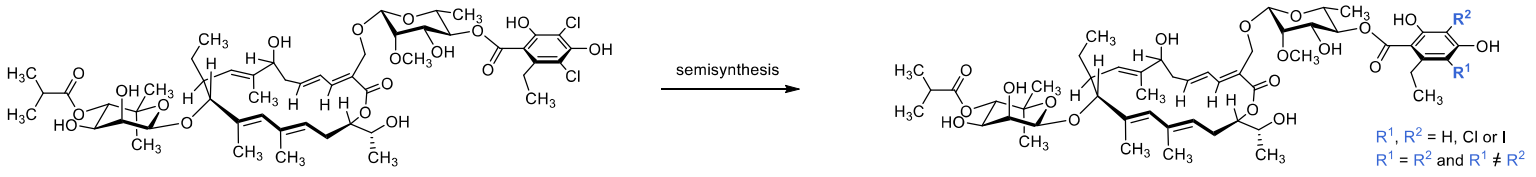

fidaxomicin (lipiarmycin A3, tiacumicin B)

\section{Twitter}

New halogenated antibiotics of the fidaxomicin class prepared @uzh_science @uzh_chemistry @karlgademann. 